\title{
Urea in the treatment of epibulbar malignancies
}

\author{
E. D. DANOPOUlOS, G. A. CHILARIS, I. E. DANOPOUlOU, and S. B. LiARigOS \\ From the Hellenic Anticancer Institute, St Savvas Hospital, Athens, Greece
}

Malignant epibulbar tumours as a rule develop in the conjunctiva, occurring most commonly at the limbus and spreading over the sclera and sometimes over the cornea. However, they can grow from the epithelium of the cornea, as in one of our cases. The usual treatment is surgical removal, as described later. The encouraging experience we had with urea treatment of primary and metastatic malignancies of the liver (Danopoulos and Danopoulou, 1974a) and of skin (Danopoulos and Danopoulou, 1974b) led us to try this treatment for epibulbar malignancy, as urea can easily be applied to the diseased area. We now report results with urea treatment in eight patients with epibulbar malignancies.

It is obvious that in cases in which the tumour has invaded the deeper layers of the globe, urea is ineffective.

\section{Patients and methods}

The patients were Greeks who were seen at the Hellenic Anticancer Institute, St Savvas, Athens, from May 1970 to February 1974. Their ages ranged from 50 to 81 years (average 65.6).

Two of the patients, both with squamous cell carcinomas, had been treated previously one with irradiation and the other with excision, and it was only after recurrence that they came to us. The first patient had a large malignant melanoma and the treatment consisted of subconjunctival injections of to per cent urea solution around the tumour. The tumour flattened after 27 injections and we then changed to instillations of the same solution 1o to 12 times daily, and the remnant of the tumour disappeared. The second patient had an extensive but flat squamous cell carcinoma of the conjunctiva and we used instillations alone with good results within 15 days. The other patients had tumorous malignancies and we decided to apply sterilized urea powder to the surface of the diseased eye, avoiding the cornea. The dilution of urea powder in the tears produces a hypertonic solution of this crystalloid, which can easily be absorbed by the conjunctiva.

\section{Method}

The head of the patient is placed in a horizontal position and eyelids are opened with a speculum. 2 to 4 drops of a 0.2 per cent novesin solution are instilled. The cornea, if it remains free of the tumour, is covered with a small Address for reprints: Professor E. D. Danopoulos MD, Rigillis Str. 26, Athens 138, Greece plastic cup to protect it from the hyperosmotic solution of urea in the tears. Half of the bulbar surface which is occupied by the tumour is then covered with sterilized urea powder. One minute later when this is diluted with tears the same powder is again added and this is repeated three to four times. Cortisone ointment is used to preverit excessive swelling of the conjunctiva and a gauze dressing applied. This sequence is repeated every second or third day depending upon the degree of irritation induced in the conjunctiva, which becomes red and oedematous. Normally five to seven treatment sessions are sufficient for the total disappearance of a tumour, although extensive tumours may require more.

Afterwards patients are advised to use instillations of 4 to 6 drops of a ro per cent urea solution in 0.9 per cent saline 10 to 12 times daily for one month, gradually decreasing them to three times daily. These instillations are not in the least irritative or painful, but they must be made for at least one year to prevent recurrence.

Two or three weeks after the end of the treatment with urea powder the conjunctiva is free of any oedema. The only undesirable effect of this treatment is an opacity of the cornea if it cannot be protected. This opacity is, however, transient and not dangerous. Two to four weeks after the course of treatment, the cornea recovers its normal appearance and the vision is re-established.

\section{Case reports}

We submit a full description of three patients, and a brief report of five patients, out of eight who had all previously suffered from epibulbar malignancies.

\section{FIRST GASE}

A 50-year-old man came to us on 17 May 1970 with a black epibulbar tumour of the left eye occupying the area between the inner canthus and limbus and protruding about $20 \mathrm{~mm}$ through the palpebral fissure (Fig. I $a$ ). The tumour had grown in 5 months from a melanosis, which had made its appearance 42 years previously after trauma from a snowball. Biopsy had shown malignant melanoma (Fig. $1 b$ ). After 27 subconjunctival injections of 2 to $3 \mathrm{ml}$ of a 10 per cent urea solution in 0.9 per cent saline, the tumour was flat (Fig. Ic) and the patient could shut his eyelids easily. Treatment was then continued with instillations Io to 12 times daily of the same urea solution. After a month the last remnants of the tumour and also the pre-existing melanosis disappeared (Fig. Id). A new biopsy was negative (Fig. $r e$ ).

The patient continued the instillations two to three times daily for 3 years, but he died $3 \frac{1}{2}$ years after the urea treat- 


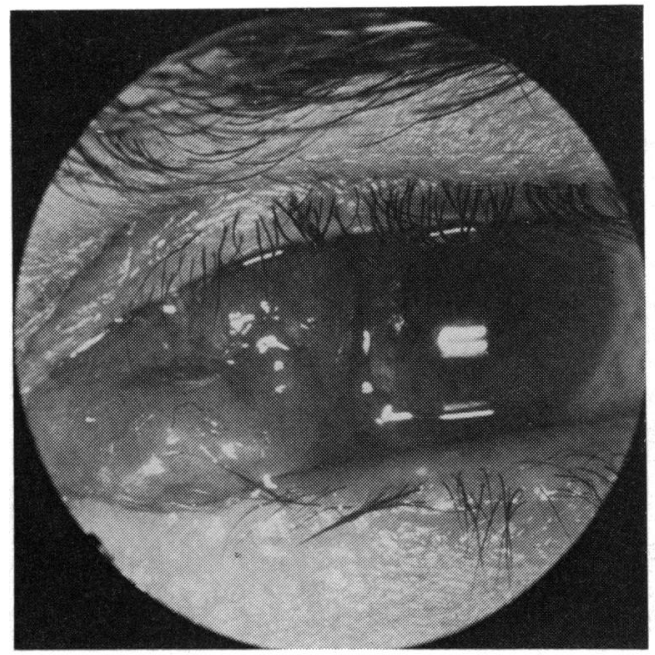

(a)
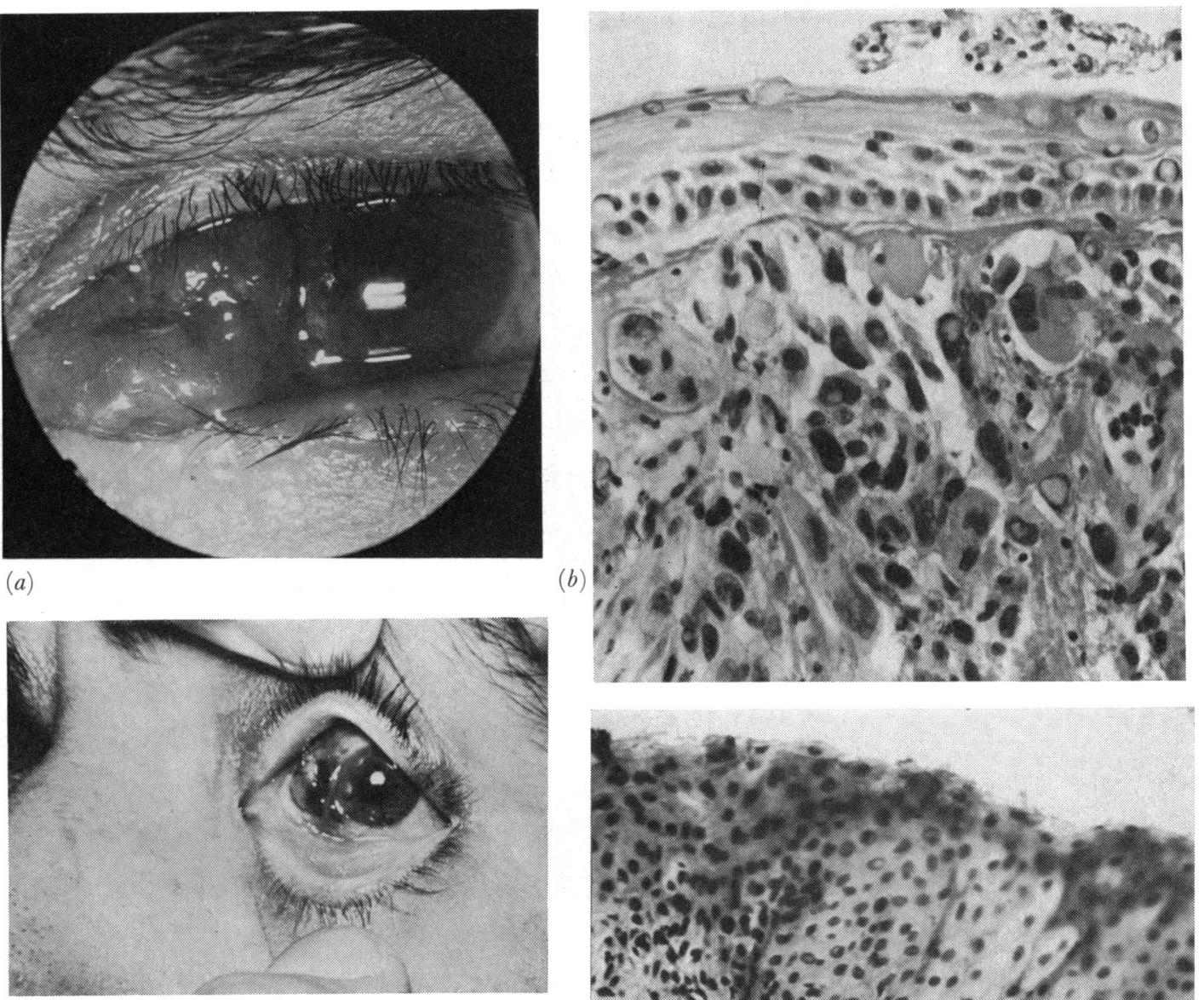

(c)

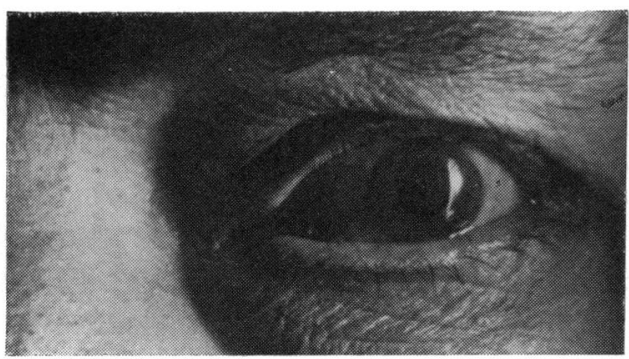

$(d)$

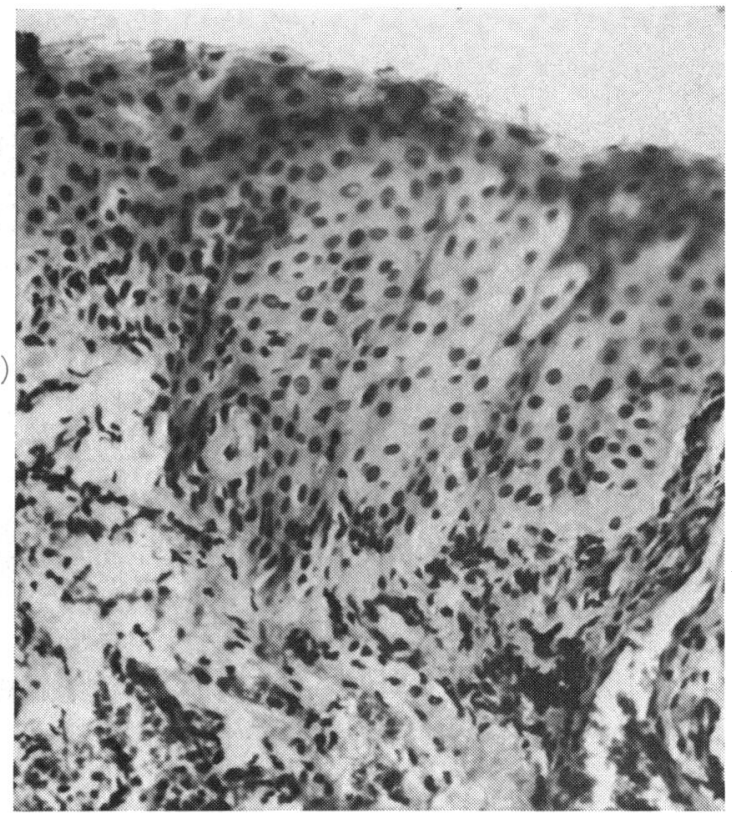

FIG. I Case I. Clinical ana histological findings (a) Malignant melanoma of conjunctiva of left eye occupying area between inner canthus and limbus (b) Histological appearance, showing malignant melanoma mostly non-pigmented.

Haematoxylin and eosin. $\times 400(c)$ Same area after 27 urea injections $(d)$ Complete healing after one month's instillations of urea solution (e) Histological appearance after treatment, showing no signs of malignancy. Haematoxylin and eosin. $\times 300$

ment from melanoma metastases without recurrence of the conjunctival primary.

SECOND CASE

A 6o-year-old woman came to us on 1o March 1973, because a thick white membrane had gradually grown in the last 3 years upon the cornea of her right eye, extending a little from the corneal pcriphery over the sclera (Fig. $2 a$ ). Over a period of 10 months the vision of the eye was lost. Biopsy showed a squamous cell carcinoma (Fig. 2b). After three treatment scssions with urea powder the 


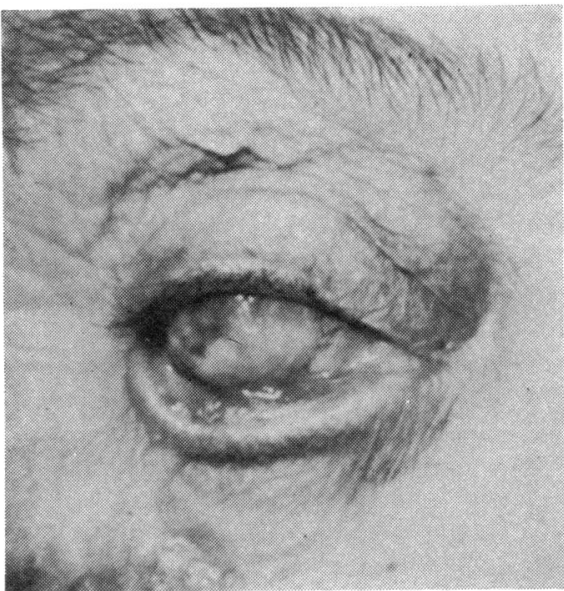

(a)

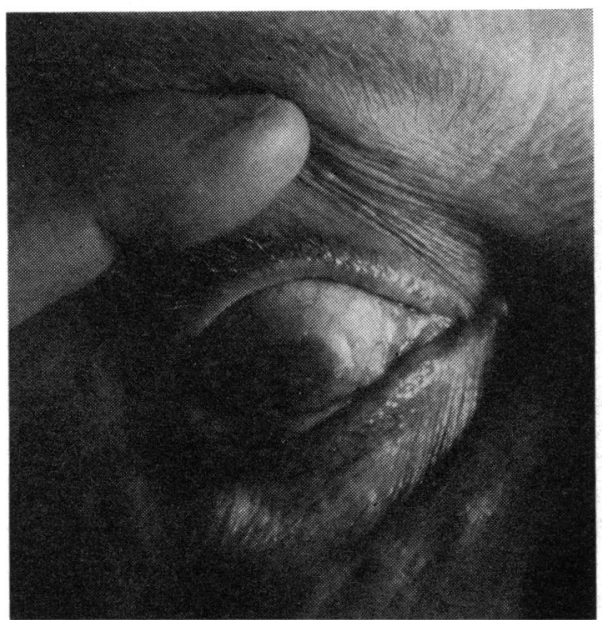

(c)
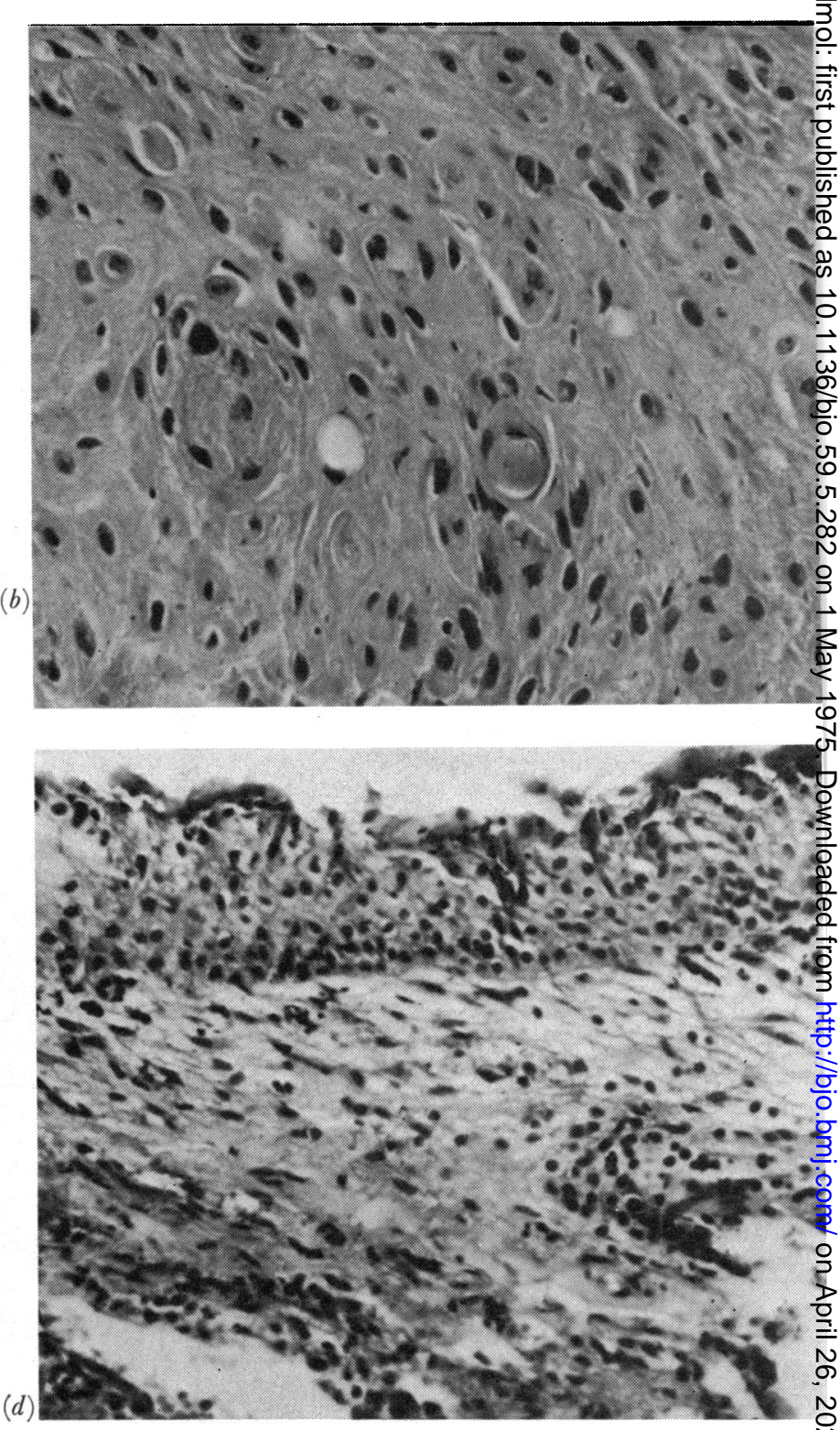

FIG. 2 Case 2. Clinical and histological findings (a) Thick white tumour occupying cornea and extending over sclera of right $\mathrm{N}_{\mathrm{J}}$ eye (b) Histological appearance, showing squamous-cell carcinoma. Haematoxylin and eosin. $\times 225$ (c) Healing after I5 treatment sessions with urea powder (d) Histological appearance after treatment, showing no signs of carcinoma. Haematoxyline and eosin. $\times 225$

patient could see again with her diseased eye. After 15 sessions the tumour disappeared but there was an opacity of the cornea which in turn disappeared after a month (Fig. 2c). A second biopsy then, and a third after 10 months were negative (Fig. $2 d$ ).

THIRD GASE

A 65-year-old woman came to us on 8 December 1973 because of a painful and very extensive whitish tumour about 2 to $3 \mathrm{~mm}$ thick, which occupied the bulbar conjunctiva from the external canthus to the limbus, $\vec{D}$ spreading and covering about two-thirds of the cornea, $\overrightarrow{\mathbb{D}}$ and extending from the superior to the inferior fornix $\varrho$ (Fig. 3a). Six months previously she had lost the sight of the diseased eye because the pupil was covered. This? tumour was a recurrence from a primary at the limbuso which had been excised one year previously. Biopsy showed a squamous cell carcinoma (Fig. $3^{b}$ ). 


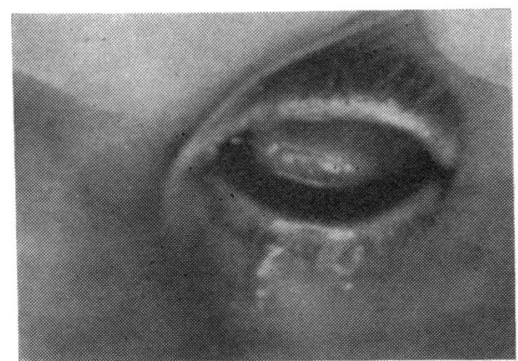

(a)

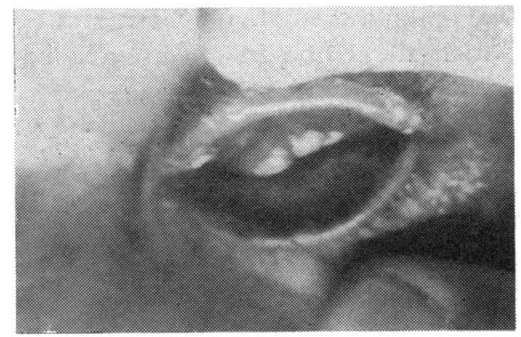

(c)

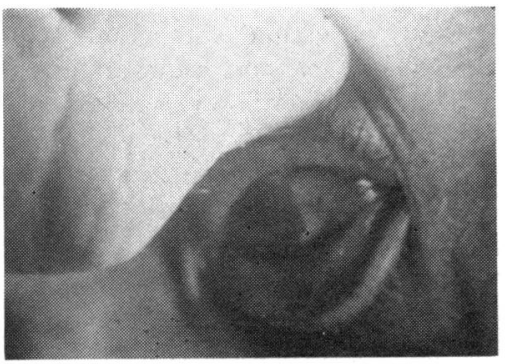

(d)
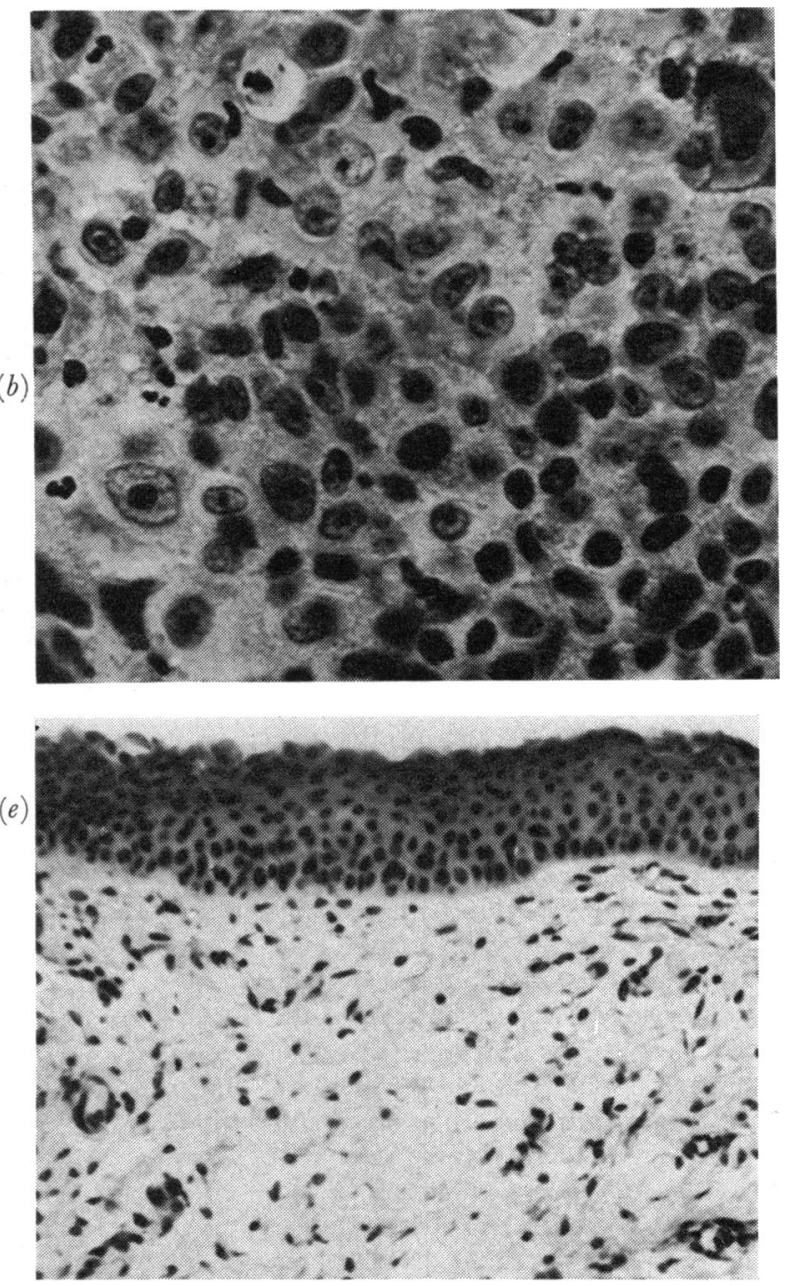

FIG. 3 Case 3. Clinical and histological findings (a) Very thick white tumour extending from superior to inferior fornix and from external canthus to limbus spreading over cornea of left eye (b) Histological appearance, showing squamous-cell carcinoma. Haematoxylin and eosin. $\times 450$ (c) Small residue of $3 \mathrm{~mm}$ diameter at limbus in $50^{\prime}$ 'clock meridian after 22 treatment sessions $(d)$ Two weeks after operative removal of small residue (e) Histological appearance after treatment, showing no signs of cancer. Haematoxylin and eosin. $\times 180$.

After 16 urea powder treatment sessions over a period of 36 days, most of the tumour mass had disappeared but there remained a small residue about $3 \mathrm{~mm}$ diameter at the limbus in the 5 o'clock meridian (Fig. $3 c$ ). After six more treatment sessions there was no change and we decided to remove it surgically because the conjunctiva had become very oedematous and the cornea opaque. Fifteen days after interrupting the urea treatment the opacity of the cornea decreased and the sight began to improve. Fifteen days later the eye and the cornea were normal (Fig. $3^{d}$ ) and the vision good. A new biopsy was negative (Fig. $3 e$ ).

The brief case reports of the other five patients cured by treatment with urea follow:
FOURTH GASE

An extensive flat squamous cell carcinoma between the limbus and the canthus (recurrence of a tumour treated by irradiation eighteen months previously) disappeared after I 5 days of instillations. The patient stopped the treatment and a recurrence appeared after one month. After a further course of one month's treatment with instillations the tumour was healed and biopsies after a month and another one after $2 \frac{1}{2}$ years were negative.

\section{FIFTH CASE}

A squamous cell carcinoma $14 \times 9 \mathrm{~mm}$ extending from 8 o'clock to 12 o'clock at the limbus was healed after only five urea powder treatment sessions. 


\section{SIXTH CASE}

A squamous cell carcinoma extending $4 \times 2 \mathrm{~mm}$ at 3.30 o'clock at the limbus was healed after only five urea powder treatment sessions.

\section{SEVENTH GASE}

A squamous cell carcinoma $15 \times 10 \mathrm{~mm}$ between 2 and 4 o'clock at the limbus and reaching the superior fornix was healed after seven urea powder treatment sessions.

EIGHTH CASE

A Kaposi tumour extending from the limbus to the external canthus was healed after 12 urea powder treatment sessions.

\section{Results}

Patients were deemed to be completely cured when the tumour had disappeared, the surface of the eye seemed normal, and the biopsy findings were negative. Except for our third patient, who had a late cure, all others were followed up for at least six months.

We think that modification of the initial urea treatment was helpful. Instead of subconjunctival injections, a sterilized powder was applied to the surface of the eye and this simplified the treatment and made it painless. Simpler still is the use of instillations, but these can be used only in cases of flat malignancies, as in the fourth case.

The only recurrence we had was our fourth case and this was because the patient stopped the instillations prematurely. The negative result of biopsy after treatment cannot exclude the possibility that in another area of the conjunctiva there still remain elements of malignancy. For this reason we advise our patients to continue instillations io to 12 times daily for at least a month after the end of treatment and a negative biopsy, and after that three times daily for at least one year. It seems unlikely that under these conditions there should be a recurrence.

In our third case, after 22 urea treatment sessions the mass of this very extensive tumour disappeared and there remained only a small residue showing a particular resistance to treatment. It would seem that urea does not act directly upon tumour tissue but rather upon the surrounding normal tissue.

Danopoulos (1970) stressed that one of the main conditions for the anticancer action of urea is that the tissue surrounding the tumour must be healthy. In this case, the conjunctiva became very oedematous as a result of numerous treatment sessions and it could not absorb urea, which was therefore ineffective. This is in agreement with our observation that in all cases most of the tumorous mass disappeared after two or three urea treatment sessions whereas regression after this becomes slower.

\section{Discussion and conclusion}

The usual methods of treatment for epibulbar malignancies are irradiation and surgery. Irradiation in the treatment of epibulbar carcinomas is generally avoided (Reese, 1966; Duke-Elder and Leigh, 1965) because of its complications (necrosis of the sclera with chronic ulcers, cataract, iridocyclitis, and secondary glaucoma).

Nevertheless Fayos and Wildermuth (1962) had good results in seven patients by giving 3380 to 4800 rads with their particular technique.

Surgery is the method normally chosen for treating these tumours. Duke-Elder and Leigh (1965) reviewing the literature and from personal experience believed that surgical excision is sufficient in the majority of epibulbar malignancies. However, in of cases in which the globe has been invaded or in or recurrences, enucleation is inevitable.

In the opinion of Reese ( 1966 ) localized tumours are easily excised. After excision of localized tumours of the limbus he advises cauterization of the limbal furrow. In cases of invasion of the sclera he advises enucleation or irradiation after excision. Sanders and Bedotto (1972) treated recurrent carcinomas in situ with wide removal of the conjunctiva with lamellar scleral and corneal resection through the corneoscleral limbus.

Eyes with extensive carcinomas are enucleated.

In 48 cases of carcinoma of the limbus Ash and Wilder (1942) found excision sufficient in 23 cases. In two cases a second operation was necessary, and in 23 cases enucleation of the globe was performed. Malignant melanomas are generally treated by exenteration of the orbit. Kaposi tumours must be treated, in the opinion of Duke-Elder and Leigh (1965), with a combination of surgical removal and irradiation.

Thus, four of our eight patients would have lost an eye if the tumour had not been treated with urea. In our first case exenteration of the orbit would have

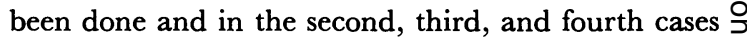
enucleation of the globe would have been indica- $\frac{D}{2}$ ted.

There is no doubt that urea treatment as described above was successful because:

(a) It did not fail in any of our eight patients although in four of them exenteration or enucleation of the globe was indicated.

(b) It is simple and can be carried out by every physician.

(c) It is usually painless after local anaesthesia with instillation of novesin.

(d) It is harmless and involves no serious side-effects. The oedema of the conjuctiva and the corneal $\stackrel{2}{\circ}$ opacity are transient.

(e) Although patients can avoid recurrences by $\varnothing$ instilling urea solution for a long period after initial treatment, recurrences may also respond to topical urea treatment. 


\section{Summary}

Eight patients with epibulbar malignancies of the eyes were successfully treated with urea.

One of them presented a malignant melanoma, one a tumour of Kaposi's disease, and six had squamous cell carcinomas.

At least four out of these eight patients would have required enucleation of the globe or exenteration of the orbit as an alternative to urea therapy.

\section{References}

ASH, J. E., and Wilder, H. C. (1942) Amer. F. Ophthal., 25, 926 DANOPOulos, E. D. (1970) Arch. Hellen. Anticancer Inst., 6, I 74 and DANOPOULOU, I. E. (1974a) Lancet, I, I 32 (1974b) Ibid., I, I 15

DUKE-ELDER, s., and LEIGH, A. G. (1965) 'System of Ophthalmology', vol. 8, p. I 144 . Kimpton, London FAYOS, J. V., and WILDERMUTH, o. (1962) Radiology, 79, 582

REESE, A. B. (1963) 'Tumors of the Eye', 2nd ed., pp. I, 316 . Hoeber, New York

SANDERS, N., and BEDotTo, c. (1972) Amer. F. Ophthal., 74, 688 